\title{
Path planning for mobile robot using the novel repulsive force algorithm
}

\author{
Yunwei Jia ${ }^{1,2, a}$, Guoqiang Yin ${ }^{1,2}$, b Lianyu Zhao ${ }^{1,2, c}$, Xueping $\mathrm{Li}^{1,2}$, Siyue \\ Sun $^{1,2}$
}

${ }^{1}$ School of Mechanical Engineering, Tianjin University of Technology, Tianjin300384, China;

${ }^{2}$ Tianjin Key Laboratory of the Design and Intelligent Control of the Advanced Mechatronical System, Tianjin 300384, China

ayunweijia@163.com, b15802270910@163.com, clianyuzhao@163.com

Corresponding Author : Guoqiang Yin

Keywords: Artificial potential field; localminimum; goal unreachable; mobile robot.

\begin{abstract}
A new type of repulsive force algorithm is proposed to solve the problem of local minimum and the target unreachable of the classic artificial potential field method in this paper. The Gaussian function that is related to the distance between the robot and the target is added to the traditional repulsive force, solving the problem of the goal unreachable with the obstacle nearby; variable coefficient is added to the repulsive force component to resize the repulsive force, which can solve the local minimum problem when the robot, the obstacle and the target point are in the same line. The effectiveness of the algorithm is verified by simulation experiments based on MATLAB.
\end{abstract}

\section{Introduction}

Robot path planning is an important part of the discipline of robotics. The idea of a path planning approach is to make the robot move from its starting point towards a destination point, while avoiding obstacles on its way [1]. There are many algorithms for robot path planning; the artificial potential field method is particularly attractive because of its mathematicalelegance and simplicity [2].The traditional artificialpotential field method assumes that the target point of the robot has a strong appeal, while obstaclesapplied repulsive force to the robot, and with the robot close to obstacles, the repulsive force willbecome increasingly large, which means that around obstacles there exists a potential field, hinderingthe robot proximity[3]. However, this method has some inherent limitations. When the target point is near the obstacle, the robot cannot reach the goal and reciprocate oscillation around the target pointbecause of the repulsion of the obstacle [4]. Due to the force balance, the robot will fall into local minimum and cannot avoid obstacle when the robot, the obstacle and the target point are in the same line [5].To overcome the above two problems, the repulsive force function is improved in this paper.

\section{The model of the APF}

\subsection{The model of the classic APF}

The repulsive potential function of the obstacle takes the following equation by Khatib[6]:

$$
\Phi= \begin{cases}\frac{1}{2} \eta\left(\frac{1}{\rho}-\frac{1}{\rho_{0}}\right)^{2} & \rho<\rho_{0} \\ 0 & \rho \geq \rho_{0}\end{cases}
$$

Where $\eta$ is a positive gaining factor, $\rho$ is the distance between the robot and theobstacle, $\rho_{0}$ is a positive constantdenoting the distance of influence of the obstacle, when $\rho<\rho_{0}$, the obstacleproduces the effect of repulsion to the robot. 
The negative gradient of the potential field is used as the virtual force acts on the robot, so the repulsive force is given by (2):

$$
\text { Frep }=-\operatorname{grad}(\Phi)=\frac{\eta}{\rho^{2}}\left(\frac{1}{\rho}-\frac{1}{\rho_{0}}\right)\left[\frac{\partial \Phi}{\partial x}, \frac{\partial \Phi}{\partial y}\right](2)
$$

The size of repulsive force is as follows:

$$
\mid \text { Frep } \mid= \begin{cases}\frac{\eta}{\rho^{2}}\left(\frac{1}{\rho}-\frac{1}{\rho_{0}}\right) & \rho<\rho_{0} \\ 0 & \rho \geq \rho_{0}\end{cases}
$$

The calculation of the angle between the robot and the obstacle is given by (4):

$$
\theta=\arccos \left(\frac{x-x_{o}}{\sqrt{\left(x-x_{o}\right)^{2}+\left(y-y_{o}\right)^{2}}}\right) \text { (4) }
$$

Where $\left[x_{o}, y_{o}\right]$ is the coordinate of the obstacle.

\subsection{The model of the modified APF}

An improved repulsive force method is proposed aiming at the two problems of the target unreachable and the local minimum in this paper. To solve the problem of the target unreachable, theGaussian function that is related to the distance between the robot and the target is added to the traditional repulsive force (5).

$$
\mid \text { Frep }\left.\right|_{\bmod i f i e d}= \begin{cases}\frac{\eta}{\rho^{2}}\left(\frac{1}{\rho}-\frac{1}{\rho_{0}}\right)\left[1-e^{-\frac{\left(x-x_{g}\right)^{2}+\left(y-y_{g}\right)^{2}}{R^{2}}}\right] & \rho<\rho_{0} \\ 0 & \rho \geq \rho_{0}\end{cases}
$$

In comparison withthe equation (3), the equation (5) solves the problem of repulsive effect at the target point which is within the distance of influence of the obstacle, ensuring the force balance of the robot. Taking into consideration the robot'sradius, it makes the path more secure and reliable at the same.

In order to make the robot escape from the local minimum, the variablecoefficients are added to the components of the repulsive forcein the $\mathrm{X}$ and $\mathrm{Y}$ axes, the equations are given by (6) and (7).

$$
\begin{aligned}
& \mid \text { FrepX } \mid=\left\{\begin{array}{lc}
(1+\alpha) \mid \text { Frep }\left.\right|_{\text {modified }} * \cos \theta & \rho<\rho_{0} \\
0 & \rho \geq \rho_{0}
\end{array}\right. \\
& \mid \text { FrepY } \mid=\left\{\begin{array}{lc}
(1+\beta) \mid \text { Frep }\left.\right|_{\text {modified }} * \sin \theta & \rho<\rho_{0} \\
0 & \rho \geq \rho_{0}
\end{array}\right.
\end{aligned}
$$

Where $\alpha$ and $\beta$ are the constants of -1 to 1 , (6) and (7) are designed to change the direction of the repulsive force through altering the size of the repulsive in the $\mathrm{X}$ axis and the $\mathrm{Y}$ axis. Such a change is able to transfer the direction of the robot's resultant force, leading the robot to march to the target and escape the local minimum point avoiding the obstacles. It can be concluded that $\alpha$ and $\beta$ should not be given the same value, otherwise, the direction of the repulsive forcewill not be changed and the robot cannot escape from the local minimum.

\section{Simulation}

In order to verify the effectiveness of the algorithm, this paper uses MATLAB to carry out the simulation experiment.

The position of the goal is $(50,50)$,the obstacles are $(15,18), \quad(30,35)$, (52,53),the distance of influence of obstacle $\rho_{0}=30, \eta=300$, the radius of the robot is $R=8$. It can be seen from Fig. 1 that the modified repulsive force algorithm has solved the problem of goal unreachable; the robot is 
not affected by the obstacle $(52,53)$.

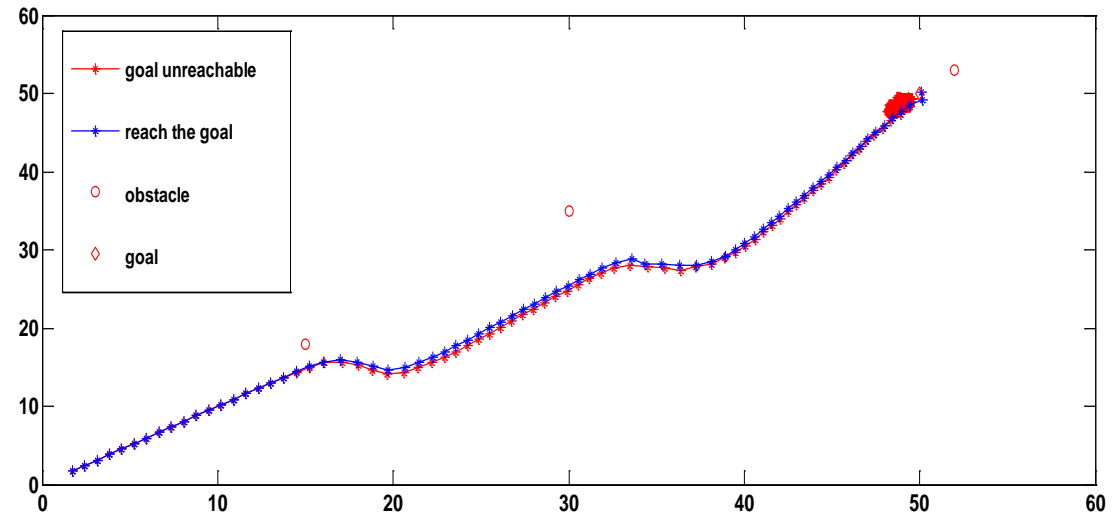

Fig.1 solved the goal unreachable

Take the variable coefficients $\alpha=-0.8 ; \beta=0.8$ the obstacles are(15,15), (20,25), (30,35),other parameters are same as the Fig.1. It can be seen from Fig.2 that the robot escapes from local minimum after add the coefficients in thecomponents of the repulsive force.

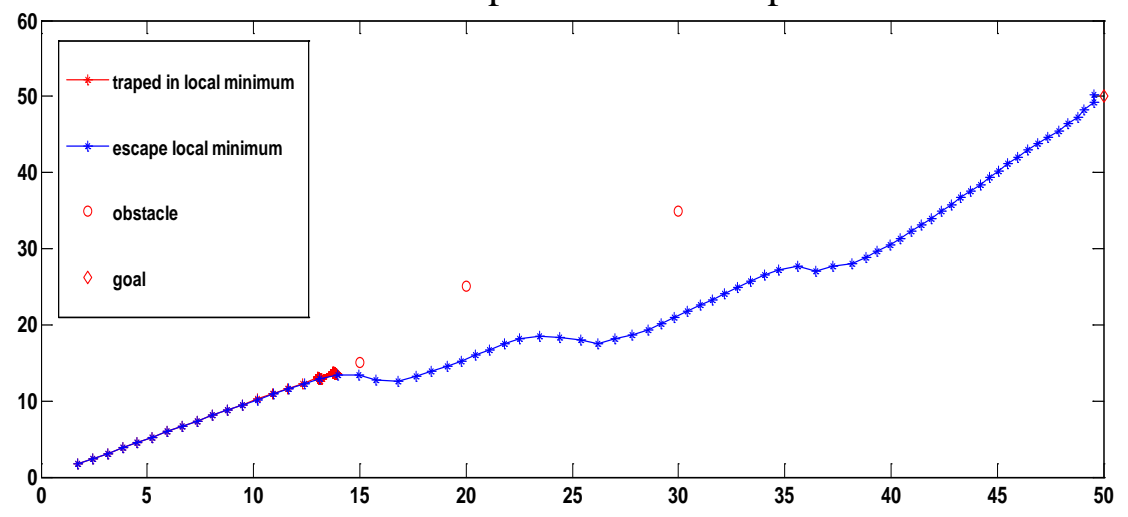

Fig.2 escape from local minimum

Set the obstacles coordinates are(15,15),(20,25)(30,35),(40,40),(52,53),other parameters are the same as above.

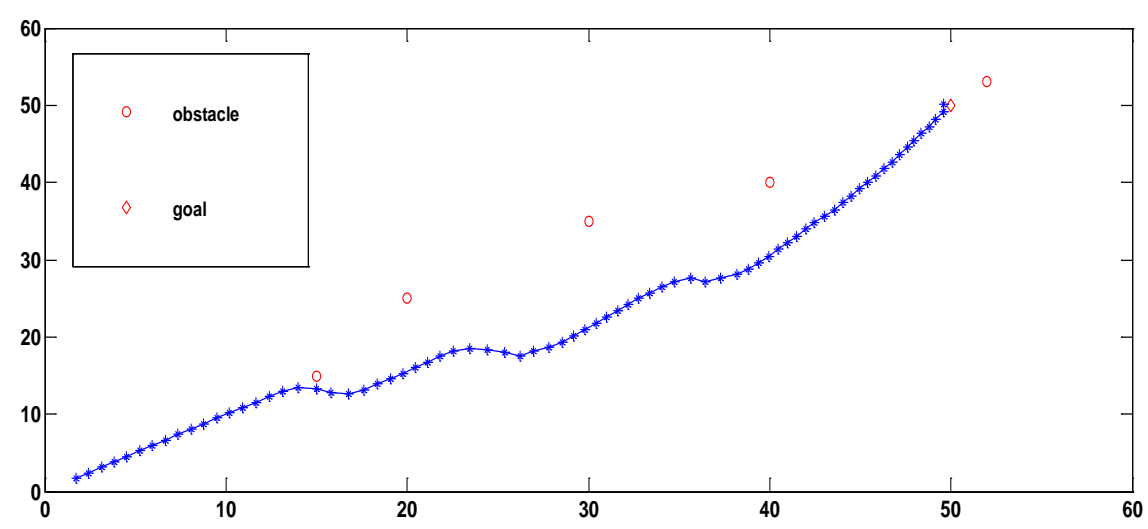

Fig.3 Path planning in two cases

In the Fig.3, the two cases of the problems of the local minimum and the goal unreachable are overcome by the improved repulsive method. After the robot escaped from the local minimum point, the robot continues to getcloser to the target and eventually stopped at the goal without the influence of the obstacle.

Set $\alpha=-0.5, \beta=0.5$ other parameters are the same as Fig.3; the path is as Fig.4. 


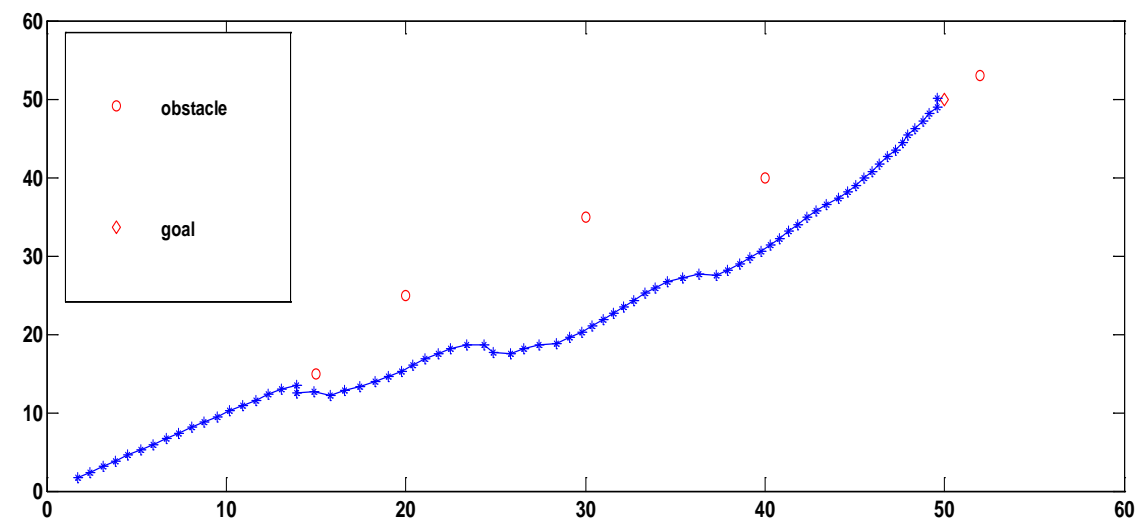

Fig.4 Path of different variables

In comparison with Fig.3, Fig.4 indicates when the variable coefficients are changed; the smoothness of the path in which the robot escapes from local minimum is also changing.

\section{Summary}

Through the simulation experiments, it can be observed that when the target is in the range of influence of the obstacle; the proposed repulsive algorithmavoids the robot to beaffected by the obstacle and solves the problem of goal unreachable. Moreover, by changing the size of the repulsive force in the $\mathrm{X}$ axis and the $\mathrm{Y}$ axis, we can overcome the problem of the local minimumon the condition that the robot, the obstacle and the target are at the same line. In addition, it canbe seen from the Figure 3 and Figure 4 thatthe values of the variables that are added to the components of the repulsive force will affect the escaping path from the local minimum of the robot, if the value is not appropriate, the path of the robot mayhave a mutation and lead easily to an oscillation.

\section{Acknowledgement}

National Natural Science Foundation of China (No.61201081), Science and Technology Development Foundation of University of Tianjin (NO. 20130704)

\section{References}

[1].Jinchao Guo, Yu Gao, Guangzhao Cui.Path planning of mobile robot based on improved potential field. Information Technology Journal. Vol.12 (2013) NO.11, p.2188-2194.

[2].S.S.Ge, Y.J.Cui. New Potential Functions for Mobile Robot Path Planning. J.IEEE Transactions on robotics and automation.2000, p.615-620.

[3].Hao Wang, Lianyu Zhao, Wei Chen. A Mobile Robot Obstacle Avoidance Method based on ImprovedPotential Field Method. Applied Mechanics and Materials. Vol.467 (2014), p.496-501.

[4]. J.Sfeir, M.Saad, H.Saliah-Hassane. An improved artificial potential field approachto real-time Mobile robot path planning in anUnknown Environment. IEEE International Symposium on Robotic and Sensors Environments .2011, p. 208-213.

[5].Li Zhou, Wei Li. Adaptive artificial potential field approach for obstacle avoidance path planning. 2014 Seventh International Symposium on Computational Intelligence and Design.p.429-432.

[6].O.Khatib. Real time obstacle avoidance for manipulators and mobile robots. IEEE International conference on Robotics and Automation. 1985, p.500-505 